\title{
Exploiting trap color to improve surveys of longhorn beetles
}

\author{
Giacomo Cavaletto $^{1} \cdot$ Massimo Faccoli $^{1} \cdot$ Lorenzo Marini $^{1} \cdot$ Johannes Spaethe $^{2} \cdot$ Filippo Giannone $^{3} \cdot$ Simone Moino $^{1}$. \\ Davide Rassati ${ }^{1}$ [e
}

Received: 6 May 2020 / Revised: 12 November 2020 / Accepted: 17 November 2020 / Published online: 7 December 2020

(c) The Author(s) 2020

\begin{abstract}
Longhorn beetles are commonly moved among continents within wood packaging materials used in trades. Visual inspections carried out at points of entry often fail to detect exotic longhorn beetles as infested materials may have little or no sign of colonization. Black-colored traps baited with pheromones and host volatiles are thus used to improve chances of detection. Here we tested whether existing surveillance protocols for longhorn beetles can be further improved using trap colors different than black. Baited traps of eight different colors (i.e., grey, yellow, green, red, blue, brown, purple and black) were deployed in a randomized complete block design at 16 sites in northern Italy in 2019. A total of 6,001 individuals from 56 longhorn beetle species were trapped. In general, yellow and blue traps caught a significantly higher number of longhorn beetle species than black traps. In addition, trap color significantly affected species richness and abundance at the subfamily and species level, with mixed response mostly linked to the habit of visiting flowers for food. Flower-visiting longhorn beetles mainly exhibited clear preference for flower-related colors, i.e., yellow, green and blue, whereas non-flower-visiting species were more attracted by dark and long-wavelength-dominated colors, like red and brown. Our results clearly indicate that generic surveillance programs should not rely exclusively on black traps and that the use of more trap colors can strongly improve the chance of detecting native and exotic longhorn beetles potentially moved with trades.
\end{abstract}

Keywords Cerambycidae $\cdot$ Color vision $\cdot$ Early detection $\cdot$ Exotic species $\cdot$ Surveillance

\section{Key message}

- Black-colored traps baited with attractive blends are used at ports of entry worldwide to intercept longhorn beetles accidentally introduced via international trade.

Communicated by J. J. Duan.

Electronic supplementary material The online version of this article (https://doi.org/10.1007/s10340-020-01303-w) contains supplementary material, which is available to authorized users.

Davide Rassati

davide.rassati@unipd.it

1 Department of Agronomy, Food, Natural Resources, Animals and Environment (DAFNAE), University of Padova, Viale dell' Università, 16, 35020 Legnaro, PD, Italy

2 Department of Behavioral Physiology \& Sociobiology, Biozentrum, University of Würzburg, Am Hubland, 97074 Würzburg, Germany

3 Padova, Italy
- No study thoroughly tested whether colored traps perform better than black traps in detecting longhorn beetles.

- Trap color can strongly increase attractiveness of longhorn beetles to baited traps, especially when targeting flower-visiting species.

- Surveillance programs must include colored traps along with commonly used black traps to improve the likelihood of detecting longhorn beetles potentially moved with trades.

\section{Introduction}

World trends of growing national and international trade have led to increased risk of native insect range expansion and exotic insect introductions (Brockerhoff and Liebhold 2017). Among the most frequently intercepted insect groups are wood-boring beetles in the family Cerambycidae (Eyre and Haack 2017). These insects, also known as longhorn beetles, are commonly moved at the larval stage within fresh 
wood packaging materials, round wood and planting material (Wu et al. 2017; Meurisse et al. 2019). Woody materials or live plants containing early instar larvae often have few or no outward signs of infestation (Humble 2010); thus, routine visual inspections carried out at points of entry often fail to detect accidentally introduced species. In addition, exotic longhorn beetles can cause massive economic and ecological damage in the invaded areas (Haack 2017) and eradication campaigns can be extremely costly (Haack et al. 2010; Faccoli and Gatto 2015). A number of phytosanitary regulations have been implemented to reduce the risk of international insect incursions (Allen et al. 2017), but several exotic longhorn beetle species continue to be detected worldwide (Eyre and Haack 2017). Thus, the availability of effective detection tools able to intercept exotic longhorn beetles before they become established in the invaded environment is essential to prevent the damage and reduce overall costs.

A number of innovative tools for early detection of exotic insects have been developed in recent years (Poland and Rassati 2019). Among them, traps baited with blends of pheromones and kairomones and placed in and around entry points represent one of the most efficient and commonly adopted approaches for longhorn beetles (Eyre and Haack 2017). Besides their low cost and ease of use, baited traps benefit from recent advances in longhorn beetle chemical ecology (Hanks and Millar 2016; Millar and Hanks 2017) and our understanding of factors that affect longhorn beetle trapping success (Allison and Redak 2017). Sex and aggregation-sex pheromones have been already identified for more than 300 longhorn beetle species worldwide (Millar and Hanks 2017), and this number is constantly growing. In addition, the effect of trap type (e.g., Graham et al. 2012), trap design (Allison et al. 2014), trap coatings (Graham and Poland 2012) as well as trap position in terms of height above the ground level (e.g., Flaherty et al. 2019; Ulyshen and Sheehan 2019; Miller et al. 2020) and distance from the forest edge (e.g., Allison et al. 2019) have been assessed for several longhorn beetle species. As a result, a wide set of trapping protocols currently exist for both species-specific and generic surveillance programs, allowing phytosanitary inspectors to select from time to time the best trapping protocol according to their needs (Poland and Rassati 2019). There is increasing evidence, however, that the efficacy of baited traps can be further increased by exploiting trap color.

Surveillance programs aimed at intercepting exotic longhorn beetles have so far relied almost exclusively on black traps (Brockerhoff et al. 2006; Bashford 2008; Rassati et al. 2015; Canadian Food Inspection Agency 2017). This practice was supported by a number of studies showing a higher efficacy of black traps compared with white or clear traps in attracting certain longhorn beetle species (De Groot and Nott 2001; Campbell and Borden 2009; Rassati et al. 2012; Kerr et al. 2017). This observation was ascribed to the resemblance of black traps to the silhouette and typical dark colors of host tree bark. Longhorn beetles, however, exploit visual stimuli not only to locate host vs. non-host plants when ovipositing (Campbell and Borden 2009; Lyu et al. 2015), but also to spot mates or feeding substrates (Monnè et al. 2017). The body color, shape and size, for example, are visual cues commonly used by adult longhorn beetles to efficiently locate mates at short-distance range (Wang 2002; Fukaya et al. 2004, 2005; Lu et al. 2007; Johnson et al. 2019). Similarly, pollen- and nectar-feeding longhorn beetles likely exploit flower colors to locate feeding substrates, although such observations exist so far only for a few species (Imrei et al. 2014; Toshova et al. 2016). It is thus reasonable to assume that colored traps might perform better than the commonly used black traps in attracting those longhorn beetle species that exploit colors as visual stimuli during at least a part of their life cycle. Trapping studies carried out so far to test this hypothesis were limited to a low number of colors, including only part of the color spectrum (Braman et al. 2003; Campbell and Hanula 2007; Shipman 2011; Skvarla and Holland 2011; Skvarla and Dowling 2017; Rassati et al. 2019), or a low number of species (Imrei et al. 2014; Toshova et al. 2016; Kerr et al. 2017), and thus, there is a clear need to expand such studies to more colors and a wider range of longhorn beetle species.

In this study, we investigated whether trap color could be exploited to improve trapping protocols for native and exotic longhorn beetles. In particular, we tested whether longhorn beetle species richness (i.e., number of species) and abundance (i.e., number of individuals) in baited traps would increase using colors different than black. Comparisons among trap colors were made following two main criteria. The first criterion was based on taxonomy (i.e., family, subfamily, and species level). A previous study showed that trap color (green vs. purple) affected longhorn beetles responses differently at the subfamily and species levels (Rassati et al. 2019); thus, we expected to observe a similar pattern in this study. We predicted that Lamiinae, many of which are nocturnal or crepuscular species that do not visit flowers (Monnè et al. 2017), prefer dark and achromatic colors, whereas we expected a clear preference for flowerlike colors in diurnally active flower-visiting species such as Lepturinae (Monnè et al. 2017). In addition, we predicted a mixed response for Cerambycinae, given that this subfamily includes species with extremely diverse biological attributes, i.e., from nocturnal species that do not visit flowers to brightly colored diurnal species that commonly visit flowers (Monnè et al. 2017). The second criterion had instead a biological basis and was selected to overcome the heterogeneity that we expected when using the taxonomic criterion. Flower-visiting insects commonly exhibit color preferences (Vuts et al. 2012; Streinzer et al. 2019), and we expected that nectar- and pollen-feeding longhorn beetle species would be 
more attracted to traps painted with the typical flower colors (e.g., yellow and blue) than to black traps, while longhorn beetle species that do not visit flowers could be efficiently trapped with the commonly used black traps.

\section{Materials and methods}

\section{Study area}

The study was conducted across 16 sites located in the Euganean Hills area, Veneto Region, northeastern Italy (Table S1; Fig. S1). This area covers about 22,000 ha and is composed of about 100 hills ranging from a few tens of meters to about $600 \mathrm{~m}$ a.s.l. in elevation. The landscape is characterized by the presence of fragmented forest patches, interspersed with various types of crop fields and urban areas. The main forest types occurring in the area are: i) chestnut (Castanea sativa Miller) forests (ca. 30\%); ii) dry forests dominated by Fraxinus ornus L. and Ostrya carpinifolia Scopoli (ca. 15\%); and iii) oak (Quercus pubescens Willd.) forests (ca. 10\%). In addition, past forest management (i.e., clear cutting) has favored the invasion of black locust (Robinia pseudoacacia L.), which now covers around $38 \%$ of the total forest area. Finally, some plantations of Austrian pine (Pinus nigra Arnold) are present (ca. 2\%). Almost all broadleaf forests are coppiced at a small spatial scale $(<0.5$ ha) with short rotation cycles (usually $<30$ years).

\section{Trap type, trap color and experimental scheme}

At each site, we used eight handmade panel traps (see Fig. S2 for details on trap design and dimensions), each painted with one of the following colors: grey, yellow, green, red, blue, brown, purple and black (corresponding to RAL numbers 7034, 1018, 6037, 3020, 5015, 8002, 4008 and 9005, respectively; Fig. S3) (Seven Colors paint factory, Sant'Angelo di Piove di Sacco, Padova, Italy). These colors were chosen because they cover a wide range of the visible spectrum. White was not included due to its low attraction toward longhorn beetles (De Groot and Nott 2001; Campbell and Borden 2009; Kerr et al. 2017). Regarding trap type, we decided to use panel traps because they are generally more effective than multi-funnel traps in trapping longhorn beetles (Allison and Redak 2017). All traps consisted of white corrugated plastic boards that were coated after painting with a $10 \%$ solution of Fluon (Insect-A-Stop, Springwood, Queensland, Australia) diluted in water. Fluon was used because it improves trapping efficacy without affecting the relative reflectance of the coated surface (Allison et al. 2016). Trap-collecting cups were half-filled with $50 \%$ solution of ethylene glycol to kill and preserve captured beetles, and the solution was replaced at each trap check. The eight treatments (i.e., colors) were replicated 16 times (i.e., sites) in a randomized complete block design, in which each block was represented by a different site (Fig. S1). At each site, traps were deployed in a linear pattern at a distance of $1.5 \mathrm{~m}$ apart. Traps were hung on a steel wire strung between two trees at about 5-7 m above the ground (Fig. S1). The latter trees were selected based on position and suitability to hold the weight of the traps, irrespective of the species. This trap height was selected because it is a good compromise to sample both longhorn beetle species active in the canopy and in the understory (Sheehan et al. 2019). In addition, traps were placed along the forest edge at each site. Traps were placed in the field from mid-May to the end of August 2019 and were checked every 3 weeks for a total of 5 times (i.e., 5 June, 26 June, 17 July, 7 August, and 28 August). All trapped longhorn beetles were identified to species using morphological features and keys (Bense 1995). Each species was then classified as either a flower-visiting or non-flowervisiting species using the Titan database (http://titan.gbif. fr/accueil_uk.html), which reports for each longhorn beetle species the scientific name of visited flowers, if any. This list was then validated and adjusted based on other available literature. Voucher specimens were deposited in the insect collection of the Entomology laboratory at the Department of Agronomy, Food, Natural Resources, Animals and Environment (DAFNAE), University of Padua (Italy).

\section{Lures}

Each trap was baited with a blend containing the primary pheromone components of the subfamily Cerambycinae and Lamiinae, which is attractive to a wide range of longhorn beetle species (Fan et al. 2019). The blend included: (i) fuscumol (volume amount: $50 \mathrm{mg}$ ); (ii) fuscumol acetate $(50 \mathrm{mg})$; (iii) geranyl acetone $(25 \mathrm{mg})$; (iv) 3-hydroxyhexan-2-one (50 mg); (v) prionic acid (1 mg); (vi) 2-methylbutan-1-ol (50 mg); (vii) anti-2,3-hexanediol (50 mg); (viii) monochamol $(50 \mathrm{mg}$ ), dissolved in isopropanol as a carrier to a total volume of $1 \mathrm{ml}$ per lure. All compounds were purchased from ChemTica Internacional, S.A. (Heredia, Costa Rica) except prionic acid (Alpha Scents Inc., West Linn, Oregon). One-milliliter aliquots of the blend were filled in glass vials with screw caps and stored at $4{ }^{\circ} \mathrm{C}$ until used. Then, at the beginning of the trapping trial and at each trap check (i.e., every three weeks), 1-ml aliquots were poured into a clear polyethylene sachet (Minigrip, $4 \times 6 \mathrm{~cm} \times 60 \mu$; Dutscher, Brumath, France) containing a cotton cylinder, which was hung in the center of the trap using a string. The release rate of the blend determined by mass loss under $20{ }^{\circ} \mathrm{C}$ conditions is estimated to $0.0263 \pm 0.002 \mathrm{~g} / \mathrm{d}$ (Fan et al. 2019). The pheromone blend was complemented with UHR (ultra-high release rate) ethanol (release rate 
of about $1.5 \mathrm{~g} / \mathrm{d}$ at $20{ }^{\circ} \mathrm{C}$; Econex, Spain), which is known to enhance the attraction of many longhorn beetle species to the above-listed pheromones (Miller et al. 2015; Collignon et al. 2016). The ethanol release device was a polylaminated aluminum-foil "blister" that was hung in the center of the trap directly below the sachet containing the pheromone blend. Ethanol was replaced only once during the trapping season based on its expected field life (i.e., 60 days).

\section{Statistical analyses}

The effects of trap color (categorical variable) on longhorn beetle species richness and abundance at the family and subfamily taxonomic level were tested using linear mixedeffect models (LMMs). The total number of species (i.e., species richness) and individuals (i.e., abundance) collected per trap color over the entire trapping season were the dependent variables. Abundance was log-transformed to satisfy the assumption of normality. Models included site as random factor to account for spatial dependence in the sampling design and were fitted with the function "Imer" in the package "Ime4" (Bates et al. 2017) implemented in R (R Core Team 2019). The same approach as described above was also used to test the effect of trap color on species richness and abundance of flower-visiting vs. non-flower-visiting longhorn beetles. However, the effect of trap color on abundance of individual longhorn beetle species was tested using generalized linear mixed-effect models (GLMMs). The number of individuals collected per trap color over the entire trapping season (negative binomial distribution) was included in the models as a dependent variable and site as a random factor. A negative binomial distribution was used because data were over-dispersed. Only species represented by at least 50 individuals were analyzed. In addition, for each species, only sites where at least 5 individuals were trapped were retained in the analysis. Models were fitted with the function "glmer.nb" in the package "Ime4" (Bates et al. 2017). Residual distribution was checked through the "DHARMa" package (Hartig 2017), which uses a simulation-based approach to create readily interpretable scaled residuals from the fitted LMM and GLMM. In all analyses, we used a priori contrasts between black (control) and the other colors instead of a post hoc multiple comparison test between all the color combinations. We planned this analysis a priori considering the number of replicates (no. of sites) according to the power of this analysis. The large number of pairwise comparisons $(n=28)$ would have required a much higher number of replicates that were not technically feasible.

\section{Results}

\section{General results}

A total of 6001 individuals from 56 longhorn beetle species were caught (Table 1). Cerambycinae was the most represented subfamily in terms of number of species (i.e., species richness) (31 species), followed by Lamiinae (13), Lepturinae (10) and Prioninae (2) (Table 1). The trend was different when considering abundance (i.e., number of individuals) of these subfamilies: Cerambycinae was again the most abundant (4419 individuals), followed by Lepturinae (642 individuals), Prioninae (501 individuals) and Lamiinae (441 individuals) (Table 1). Among Cerambycinae, $80 \%$ of individuals belonged to five species, namely the native Phymatodes testaceus (L.) $(1,218$ individuals), Nathrius brevipennis (Mulsant) (529 individuals), Chlorophorus sartor (Muller) (476 individuals), and Purpuricenus kaehleri (L.) (239 individuals), and the exotic Xylotrechus stebbingi Gahan (1,084 individuals). By contrast, 13 species were represented by less than 10 individuals each (Table 1). Lepturinae and Prioninae catches were also dominated by only a few species: Pachytodes erraticus (Dalman), Stictoleptura cordigera (Fuessly), and Rutpela maculata (Poda) comprised $90 \%$ of the lepturine individuals, whereas Prionus coriarius (L.) far exceeded the only other prionine species collected, i.e., Aegosoma scabricorne (Scopoli) (476 vs. 25 individuals). A similar pattern was found in the Lamiinae: Aegomorphus clavipes (Schrank) and Leiopus nebulosus (L.) comprised 90\% of lamiine individuals (Table 1). Moreover, 10 out of 13 lamiine species were represented by less than 10 individuals each (Table 1). Regarding the flower-visiting habit, all lepturine and 16 out of 31 cerambycine species were classified as flower-visitors (26 species), whereas both prionine, 15 out of 31 cerambycine, and all lamiine species were classified as non-flower-visitors (30 species).

\section{Effect of trap color at the family, subfamily and species level}

At the family level (i.e., Cerambycidae), both longhorn beetle species richness and abundance were significantly affected by trap color (Table S2). For species richness, the mean number of longhorn beetle species was significantly higher in both yellow and blue traps than in black traps, whereas no significant difference was found among black and the other tested colors (Table S3 and Fig. 1a). For abundance, black traps caught a significant higher mean number of individuals than both grey and green traps (Table S3 and Fig. 1b). 
Table 1 Total number of individuals trapped by each trap color

\begin{tabular}{lllllllll} 
Black Brown Red & Yellow Green & Blue & Purple & Grey & Total & Flowers \\
\hline
\end{tabular}

Cerambycinae

Anaglyptus gibbosus (Fabricius)

Anaglyptus mysticus (Linneaus)

Callimus abdominalis (Olivier)

Cerambyx cerdo (Linneaus)

Cerambyx scopolii Fuessly

Cerambyx welensii (Kuster)

Chlorophorus figuratus (Scopoli)

Chlorophorus glabromaculatus (Goeze)

Chlorophorus sartor (Muller)

Chlorophorus trifasciatus (Fabricius)

Chlorophorus varius (Muller)

Clytus arietis (Linneaus)

Clytus rhamni Germar

Gracilia minuta (Fabricius)

Nathrius brevipennis (Mulsant)

Neoclytus acuminatus (Fabricius)*

Phymatodes testaceus (Linneaus)

Plagionotus arcuatus (Linneaus)

Plagionotus detritus (Linneaus)

Poecilium alni (Linneaus)

Poecilium fasciatum (Villers)

Pseudosphegesthes cinerea (Castelnau \& Gory)

Purpuricenus kaehleri (Linneaus)

Ropalopus clavipes (Fabricius)

Ropalopus femoratus (Linneaus)

Stenopterus rufus Linneaus

Trichoferus holosericeus (Rossi)

Trichoferus pallidus (Olivier)

Xylotrechus antilope (Schonherr)

Xylotrechus arvicola (Olivier)

Xylotrechus stebbingi Gahan*

Lamiinae

Aegomorphus clavipes (Schrank)

Anaesthetis testacea (Fabricius)

Exocentrus adspersus Mulsant

Exocentrus lusitanus (Linneaus)

Exocentrus punctipennis Mulsant \& Guillebeau

$\begin{array}{llll}1 & - & - & 1\end{array}$

$\begin{array}{lllll}- & - & - & -\end{array}$

$\begin{array}{llll}2 & 1 & 1 & 5\end{array}$

$\begin{array}{llll}- & - & - & 18\end{array}$

$\begin{array}{lllll}1 & 1 & - & -\end{array}$

$\begin{array}{llll}51 & 97 & 57 & 29\end{array}$

$\begin{array}{llll}4 & 4 & 1 & 1\end{array}$

$292 \quad 183 \quad 172 \quad 65$

- $\quad-$

33

4

$-\quad-$

- $\quad-$

$-\quad-$

$8 \quad 7$

$\begin{array}{llll}11 & 18 & 8 & 32\end{array}$

$\begin{array}{llll}11 & 1 & - & 1\end{array}$

$220 \quad 184$

$199 \quad 87$

\begin{tabular}{rlllllllrl}
29 & 36 & 52 & 24 & 29 & 53 & 58 & 26 & 307 & $\mathrm{~N}$ \\
3 & 3 & 1 & 2 & 1 & 1 & - & - & 11 & $\mathrm{~N}$ \\
2 & - & 1 & - & - & - & 1 & - & 4 & $\mathrm{~N}$ \\
1 & 1 & - & - & 2 & 3 & - & 1 & 8 & $\mathrm{~N}$ \\
- & 1 & 4 & - & - & - & - & 4 & 9 & $\mathrm{~N}$ \\
9 & 16 & 10 & 5 & 15 & 11 & 10 & 12 & 88 & $\mathrm{~N}$ \\
- & - & 1 & - & 1 & 1 & - & - & 3 & $\mathrm{~N}$ \\
- & - & - & - & - & - & - & 1 & 1 & $\mathrm{~N}$ \\
- & 1 & - & - & - & - & - & 1 & 2 & $\mathrm{~N}$ \\
- & - & - & - & - & - & - & 1 & 1 & $\mathrm{~N}$ \\
- & - & 1 & - & - & - & - & 1 & 2 & $\mathrm{~N}$ \\
- & 1 & 1 & - & - & - & - & - & 2 & $\mathrm{~N}$ \\
- & - & 2 & - & - & - & - & 1 & 3 & $\mathrm{~N}$ \\
& & & & & & & & & \\
- & - & - & - & - & 1 & - & - & 1 & $\mathrm{Y}$ \\
1 & - & 2 & 12 & - & 1 & - & - & 16 & $\mathrm{Y}$ \\
2 & 1 & 12 & 106 & 48 & 31 & - & 13 & 213 & $\mathrm{Y}$ \\
\hline
\end{tabular}

$1 \quad \mathrm{Y}$

$2 \mathrm{Y}$

$10 \mathrm{Y}$

$26 \mathrm{Y}$

$3 \mathrm{~N}$

$529 \mathrm{~N}$

$25 \mathrm{~N}$

$1218 \mathrm{Y}$

$3 \mathrm{~N}$

$39 \mathrm{~N}$

$43 \mathrm{~N}$

$1 \mathrm{~N}$

$8 \mathrm{~N}$

$239 \mathrm{Y}$

$50 \quad \mathrm{Y}$

$7 \mathrm{Y}$

$75 \quad \mathrm{Y}$

$3 \mathrm{~N}$

$61 \mathrm{~N}$

$126 \mathrm{~N}$

$5 \mathrm{Y}$

$1084 \mathrm{~N}$
Leiopus nebulosus (Linneaus)

Mesosa curculionoides (Linneaus)

Mesosa nebulosa (Fabricius)

Monochamus galloprovincialis (Olivier)

Oberea linearis (Linneaus)

Parmena unifasciata (Rossi)

Pogonocherus hispidus (Linneaus)

Saperda punctata (Linneaus)

Lepturinae

Anoplodera sexguttata (Fabricius)

Grammoptera ruficornis (Fabricius)

Pachytodes erraticus (Dalman)

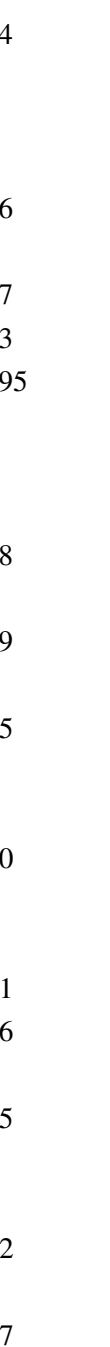

$\begin{array}{llll}9 & 7 & 10 & 9 \\ - & 1 & - & 1 \\ - & - & - & 1 \\ 1 & 1 & - & - \\ 9 & 10 & 10 & \\ - & - & - & - \\ 12 & - & - & 2 \\ 15 & 14 & 15 & \\ 75 & 3 & - & \\ - & - & - & - \\ 1 & 1 & - & - \\ - & - & 1 & - \\ 6 & 1 & 1 & - \\ - & - & - & 14 \\ 93 & 59 & 77 & 66 \\ - & 6 & 4 & 5 \\ 38 & 174 & 197 & 97\end{array}$

9
1
1
-
13
-
2
14
1
-
-
-
-
1
66
5
97

$87 \quad Y$

7 Y

$6 \quad \mathrm{Y}$

$3 \mathrm{~N}$

$94 \mathrm{Y}$

$3 \mathrm{~N}$

$32 \mathrm{Y}$

$153 \mathrm{~N}$

$476 \quad \mathrm{Y}$ 
Table 1 (continued)

\begin{tabular}{|c|c|c|c|c|c|c|c|c|c|c|}
\hline & Black & Brown & Red & Yellow & Green & Blue & Purple & Grey & Total & Flowers \\
\hline Paracorymbia fulva (De Geer) & - & - & - & - & - & 1 & - & - & 1 & $\mathrm{Y}$ \\
\hline Rutpela maculata (Poda) & 3 & 12 & 15 & 73 & 42 & 8 & 2 & 21 & 176 & Y \\
\hline Stenurella bifasciata (Muller) & - & - & 1 & 3 & 1 & 3 & - & - & 8 & $\mathrm{Y}$ \\
\hline Stenurella melanura (Linneaus) & 1 & - & 1 & 3 & - & 6 & - & 3 & 16 & $\mathrm{Y}$ \\
\hline Stenurella septempunctata (Fabricius) & 1 & 1 & - & 1 & 1 & 1 & - & - & 5 & $\mathrm{Y}$ \\
\hline Stictoleptura cordigera (Fuessly) & 2 & 2 & 4 & 9 & 2 & 161 & 10 & 7 & 197 & $\mathrm{Y}$ \\
\hline Stictoleptura scutellata (Fabricius) & 2 & - & - & - & - & 4 & 1 & 2 & 9 & $\mathrm{Y}$ \\
\hline \multicolumn{11}{|l|}{ Prioninae } \\
\hline Aegosoma scabricorne (Scopoli) & 4 & 2 & 4 & 2 & 5 & 4 & 3 & 1 & 25 & $\mathrm{~N}$ \\
\hline Prionus coriarius (Linneaus) & 79 & 60 & 154 & 32 & 28 & 38 & 51 & 34 & 476 & $\mathrm{~N}$ \\
\hline
\end{tabular}

The column "Flowers" indicates whether a given species is known to be a flower-visiting (Y) or non-flower-visiting (N) species. Species are listed alphabetically within each subfamily

*Indicate species exotic to Italy

At the subfamily level, trap color affected species richness of Cerambycinae, Lamiinae and Lepturinae (Table S2). The subfamily Prioninae was not analyzed because it was represented by only two species. For Cerambycinae, yellow traps caught significantly more species than black traps, but no difference was found between black and the other tested colors (Table S3 and Fig. 1c). For Lamiinae, both brown and red traps caught significantly more species than black traps (Table S3 and Fig. 1e). For Lepturinae, three colors attracted significantly more species than black, which were yellow, blue and green (Table S3 and Fig. 1g). Trap color also significantly affected the abundance of the three subfamilies analyzed (Table S2). For Cerambycinae, the mean number of individuals was significantly higher in black than in green, grey and blue traps (Table S3 and Fig. 1d). For Lamiinae, the mean number of individuals was higher in both red and blue traps compared with black traps (Table S3 and Fig. 1f). For Lepturinae, yellow, green, blue, red and grey traps caught significantly higher number of individuals than black traps (Table S3 and Fig. 1h).

At the lowest taxonomic level tested (i.e., individual species), 18 longhorn beetle species were represented by at least 50 individuals and thus were retained for analysis. Trap color significantly affected 13 of these species (Table S2). Five out of the eight analyzed cerambycine species were collected in significantly higher number by trap colors different than black. Cerambyx scopolii Fuessly, C. sartor, Stenopterus rufus L. and Xylotrechus antilope (Schonherr) showed a preference for yellow traps, although other colors also appeared to be more efficient than black (e.g., green for $C$. sartor and S. rufus) (Table S4 and Fig. 2). N. brevipennis showed instead a preference for brown and green traps. Black was more efficient than other colors for the native P. testaceus and the exotic $X$. stebbingi (Table $\mathrm{S} 4$ and Fig. 2). Also, the Lamiinae $A$. clavipes was caught in higher numbers by trap colors different than black, that are red, blue and purple. Among Lepturinae, $P$. erraticus and $R$. maculata were mainly caught in yellow and green traps, although other trap colors were also more efficient than black traps, whereas $S$. cordigera showed a preference for blue traps (Table S4 and Fig. 2). Finally, among Prioninae, P. coriarius was efficiently trapped with black traps, which showed a higher efficiency than green, yellow, blue and grey traps (Table S4 and Fig. 2).

\section{Effect of trap color on flower-visiting versus non-flower-visiting longhorn beetles}

Trap color affected species richness of both flower-visiting $\left(\chi^{2}=88.33 ; P<0.001\right)$ and non-flower-visiting longhorn beetles $\left(\chi^{2}=14.27 ; P=0.046\right)$, but the effect was different between these two groups. For flower-visiting longhorn beetles, the number of species was significantly higher in yellow, blue and green traps than in black traps (Table S5 and Fig. 3a), whereas yellow traps and green traps were significantly less efficient than black traps for non-flowervisiting species (Table S5 and Fig. 3c). Trap color also affected the abundance of both groups $\left(\chi^{2}=47.78\right.$ and $P<0.001$ for flower-visiting; $\chi^{2}=44.61$ and $P<0.001$

Fig. 1 Effect of trap color on longhorn beetle species richness and abundance at the family (A-B) and subfamily levels $(\mathrm{C}-\mathrm{H})$. Within each panel, colors that attracted a significantly different mean number of species or individuals than black traps are indicated with black asterisk/s or black circle depending on the $P$ value: $* * *=P<0.001$; $* *=P<0.01 ; * P<0.05$; filled circle $=P<0.1$. Abundance at the family and subfamily level are log-transformed. Error bars indicate the $95 \%$ confidence intervals for the mean 

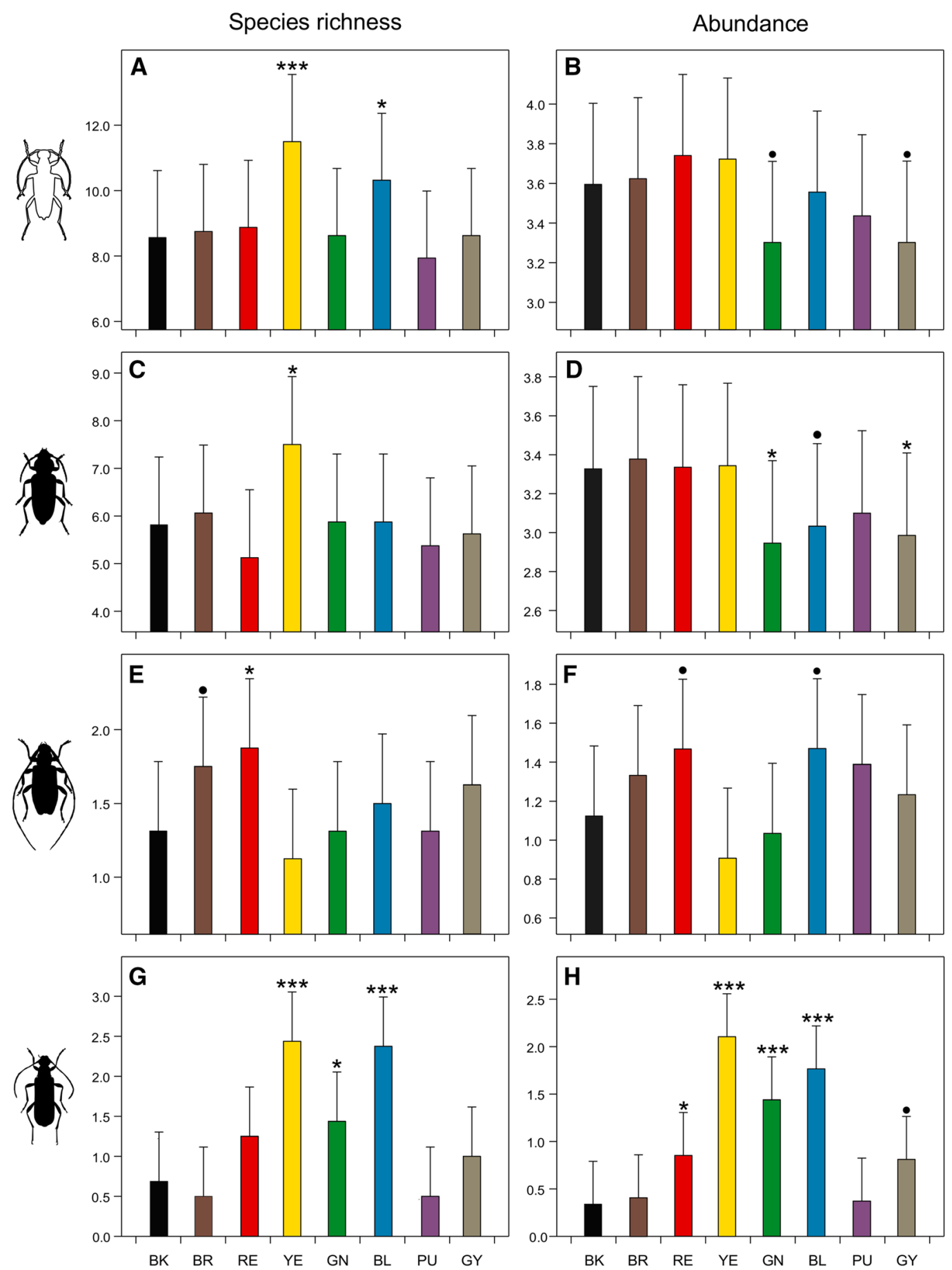

Taxonomic leve

Trap colors
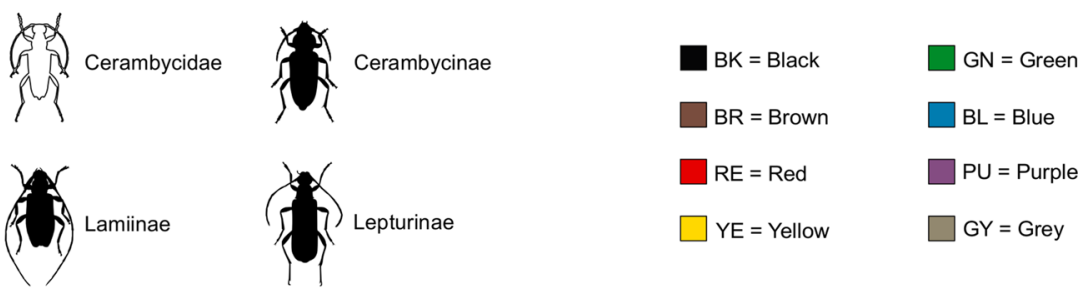

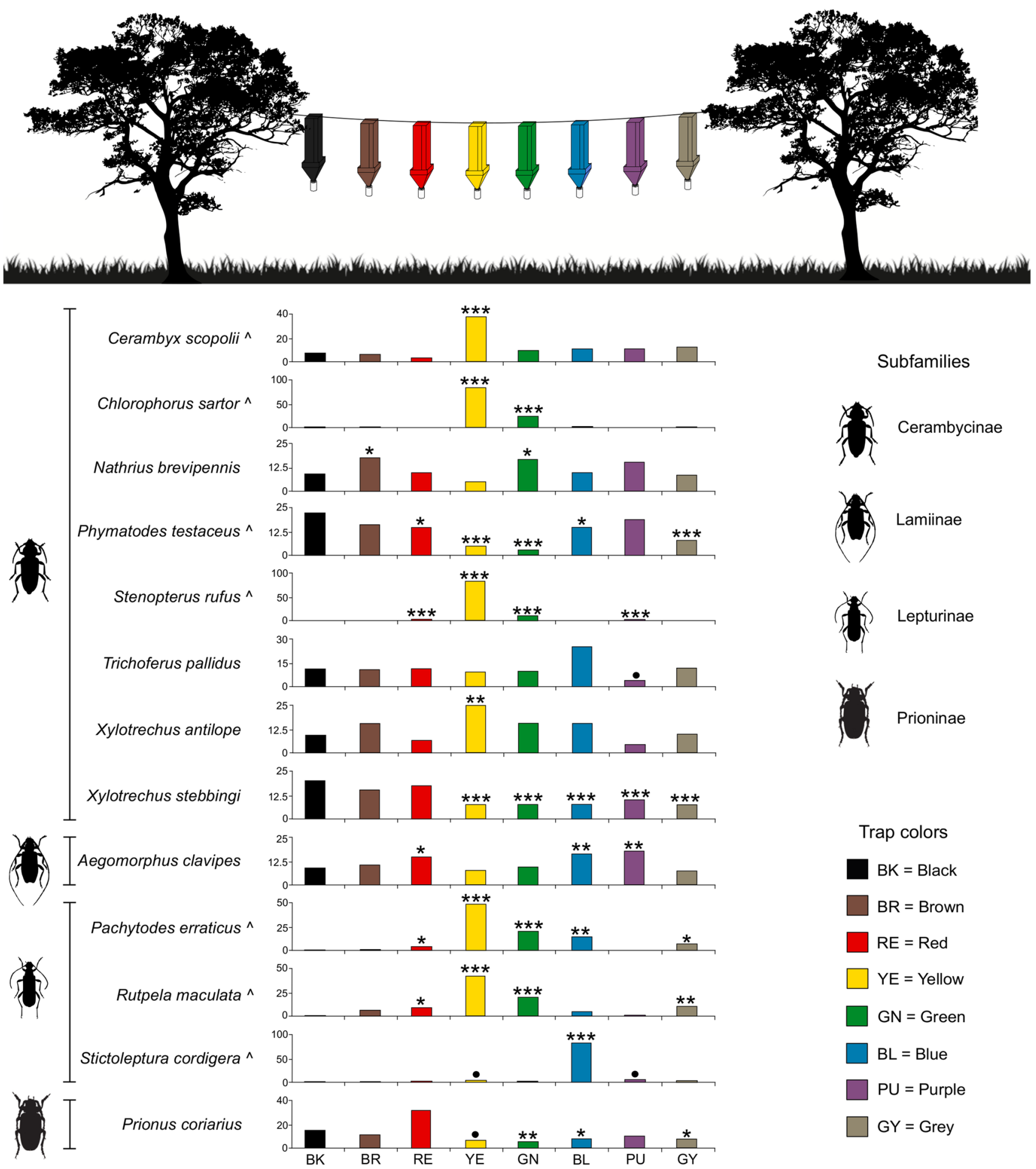

Subfamilies

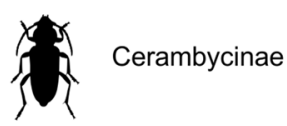

(1) Laminae

Lepturinae

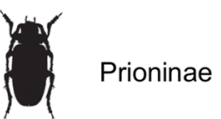

Trap colors

$\mathrm{BK}=$ Black

$\mathrm{BR}=$ Brown

$\mathrm{RE}=\mathrm{Red}$

YE $=$ Yellow

$\mathrm{GN}=$ Green

$\mathrm{BL}=$ Blue

$\mathrm{PU}=$ Purple

$\mathrm{GY}=$ Grey

Fig. 2 Proportion of individuals collected in traps painted with the eight tested colors. Data are shown only for longhorn beetle species that were significantly affected by trap color (Table S4) and are presented in alphabetical order within each subfamily. An ^ after a species name indicates flower-visiting species. Within each bar plot, colors that attracted a significantly different mean number of individuals than black traps are indicated with black asterisk/s or black circle depending on the $P$ value: $* * *=P<0.001 ; * *=P<0.01 ; * P<0.05$; filled circle $=P<0.1$ 

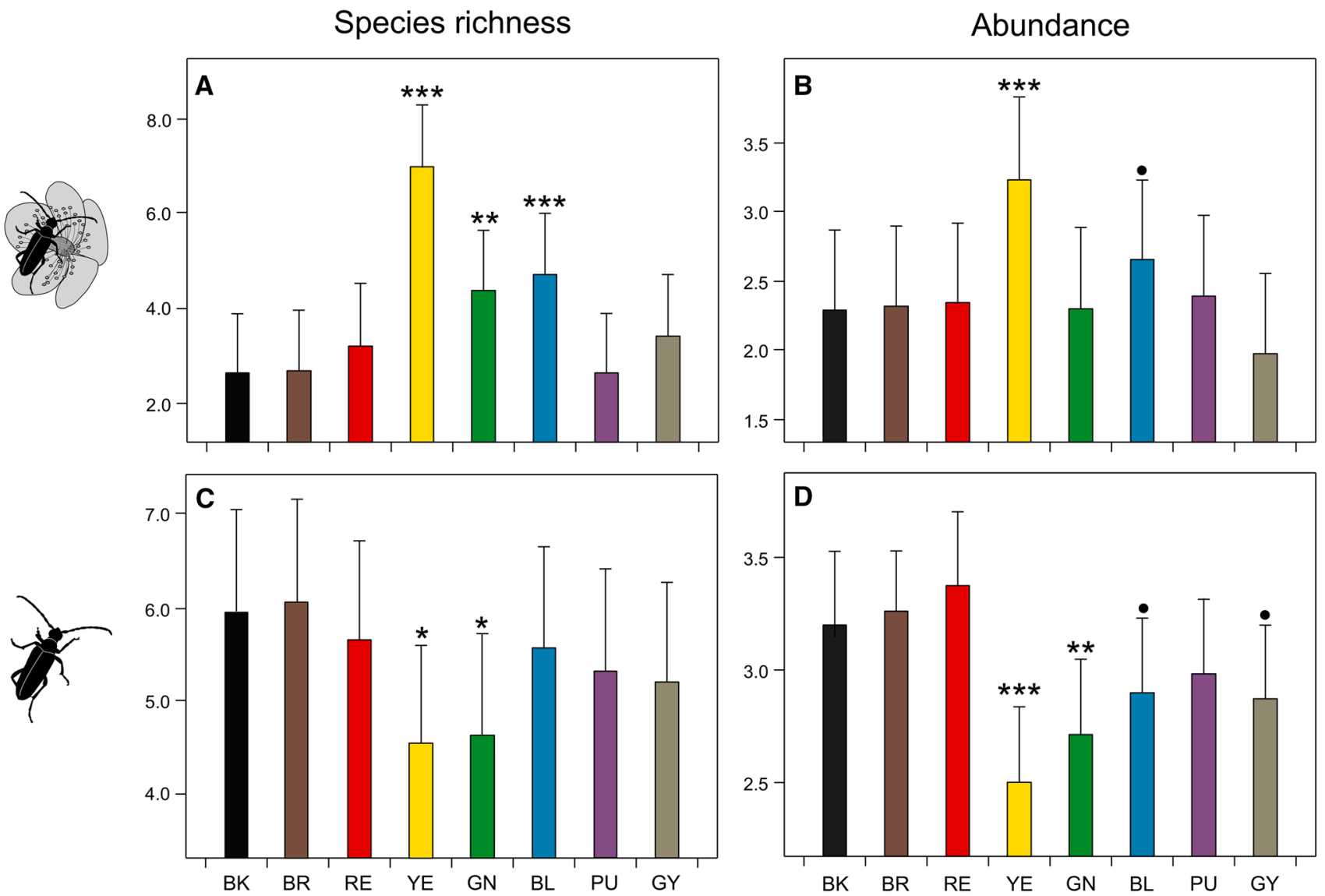

Feeding behaviour

Flower-visitors

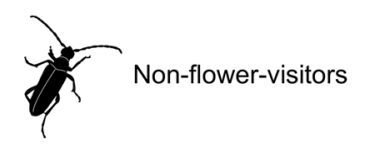

Fig. 3 Effect of trap color on species richness and abundance of flower-visiting (A, B) vs. non-flower-visiting (C, D) longhorn beetles. Only significant results are shown. Within each panel, colors that attracted a significantly different mean number of species or individuals than black traps are indicated with black asterisk/s or black circle

for non-flower-visiting). The mean number of individuals of flower-visiting species was significantly higher in yellow traps and blue traps than in black traps (Table S5 and Fig. 3b), whereas the opposite trend occurred for nonflower-visiting species, with yellow traps, along with green, blue and grey traps, catching significantly lower numbers of individuals than black traps (Table S5 and Fig. 3d). depending on the $P$ value: $* * *=P<0.001 ; * *=P<0.01 ; * P<0.05$; filled circle $=P<0.1$. Abundance of both flower-visitors and nonflower-visitors are log-transformed. Error bars indicate the 95\% confidence intervals for the mean

\section{Discussion}

Surveillance programs for longhorn beetles commonly exploit traps baited with pheromones and host volatiles placed in and around entry points. However, most of these programs rely almost exclusively on black-colored traps. Here, we showed that trap color can strongly affect longhorn beetle species richness and abundance in traps, with mixed responses at subfamily and species level. In addition, we showed that the response of longhorn beetles to trap color appears to be linked to their ecology, especially 
the habit of visiting flowers for food. Our results provide evidence that trap color should be considered an important variable when planning surveillance programs for longhorn beetles and that the use of colored traps can strongly improve the likelihood of intercepting native and exotic species moved with national and international trade.

Trap color significantly affected longhorn beetle species richness at the family level. Yellow and blue traps caught a significantly higher number of species than black traps, clearly indicating that generic surveillance programs should not rely exclusively on black traps. The use of black traps is based on studies showing that black or dark colors are more effective than white, transparent or clear traps in attracting longhorn beetles (De Groot and Nott 2001; Campbell and Borden 2009; Rassati et al. 2012; Kerr et al. 2017). However, most of these studies targeted individual species, and the higher efficacy of black vs. clear traps was not supported by analyses carried out at the family level (Bouget et al. 2008; Allison and Redak 2017). The option of using colored traps was rarely considered in the past, likely because indications that colors different than black can efficiently attract longhorn beetles were controversial and based on comparisons among a limited number of colors (Shipman 2011; Skvarla and Holland 2011; Skvarla and Dowling 2017; Rassati et al. 2019). Thus, our results provide the first evidence that colored traps can strongly increase the overall attractiveness of longhorn beetles to baited traps. This pattern was not valid for abundance, probably due to a high variation of preferences among species that leveled out at family level.

Trap color significantly affected longhorn beetle species richness and abundance also at the subfamily level, but the effect differed between subfamilies. A previous study showed that Cerambycinae and Lamiinae were differentially attracted by purple and green traps (Rassati et al. 2019), a trend that was attributed to the different flight activity patterns (nocturnal vs. diurnal) of the main representatives of the two subfamilies. Our results confirmed that the biological attributes shared by most longhorn beetle species within a given subfamily affect their response to trap colors. Lepturinae, most of which are diurnally active and flower-visitors (Monnè et al. 2017), exhibited clear preference for flower-related colors, i.e., yellow, green and blue, over black. In contrast, Lamiinae, of which many are nocturnal or crepuscular species and do not visit flowers (Monnè et al. 2017), were more attracted by dark and longwavelength-dominated colors, like red and brown. Cerambycinae, which include both diurnal flower-visiting species and nocturnal non-flower-visiting species (Monnè et al. 2017), showed a less clear pattern, with large discrepancies between trends for species richness (i.e., yellow traps more efficient than black traps) and abundance (i.e., black traps more efficient than green and grey traps).
At the species level, 13 longhorn beetle species were significantly affected by trap color. Nonetheless, patterns among these species were found to be quite heterogeneous and only partially generalizable using taxonomical or biological criteria. Within the subfamily Cerambycinae, for example, the exotic $X$. stebbingi showed a clear preference for black over other colors, similar to what was found by Skvarla and Dowling (2017) for Xylotrechus colonus (Fabricius), indicating that choice behavior was largely guided by achromatic vision. Xylotrechus antilope, instead, showed a different pattern, with a strong preference for yellow traps over black traps, despite the fact that both of these Xylotrechus species are non-flower-visitors. Within the flower-visitors, $R$. maculata and S. cordigera showed clear preferences for yellow/green and blue over black, respectively. The difference in color preference might be linked either to the colors of the most commonly visited flowers or to differences in the (visual guided) mate-finding behavior. X. stebbingi and X. colonus, for example, are mainly crepuscular or nocturnal species (Rassati et al. 2020), whereas $X$. antilope is mainly diurnal, with maximal activity in the late morning to early afternoon (Molander et al. 2019). This difference is also reflected in their body coloration: $X$. antilope is black with yellow stripes and likely mimic vespid wasps (Mitchell et al. 2017), $X$. stebbing $i$ and $X$. colonus are reddish-grayish, which likely allow them to camouflage when moving on tree bark. It is thus likely that these longhorn beetle species exploit different visual channels (i.e., acromatic in $X$. stebbingi and $X$. colonus and chromatic in $X$. antilope), representing an adaptation to their diel activity pattern. The preference of $X$. antilope for yellow may be linked to an innate preference for the yellow color present on their body and thus a mechanism involved in mate location. $R$. maculata and $S$. cordigera are both flower-visitors, but the range of visited flowers varies between them. Flower color is one of the most important features used by flower-visiting insects to locate flowers and discriminate between plant species (Gumbert 2000; Streinzer et al. 2019), and it is likely that $R$. maculata and $S$. cordigera have innate preference for colors that correspond to those of the most commonly visited flowers. Further studies are, however, needed to elucidate these mechanisms.

Both species richness and abundance of flower-visiting and non-flower-visiting longhorn beetles were affected by trap color, but with opposite patterns. Colors that attracted a higher number of flower-visiting species and individuals than black traps (i.e., yellow, green and blue) resulted in being less efficient than black traps for non-flower-visiting longhorn beetles and vice versa. The ability to discriminate among different colors and an innate preference for blue or yellow is well documented in flower-visiting insects (e.g., Giurfa et al. 1995; Gumbert 2000; Schaefer et al. 2004; 
Balamurali et al. 2019), but only a few observations have been reported so far in longhorn beetles (Imrei et al. 2014; Toshova et al. 2016), despite the presence of several spectrally different photoreceptor types found in Coleoptera (Sharkey et al. 2017). Our results provide evidence that flower-visiting longhorn beetles are able to distinguish color, guided by a chromatic visual channel, and indicate that color is exploited to locate feeding substrates in addition to locating potential mate (Johnson et al. 2019). Most non-flowervisiting longhorn beetles feed on twig bark or tree foliage, or do not feed at all (Monnè et al. 2017), and it is thus not surprising that such beetles are not attracted by typical flower colors. For the latter longhorn beetles, choosing the darkest colors (in our case black, brown and red) might be a proper mechanism to distinguish dark objects (e.g., the tree trunk) against bright background (sky), as well as between bark of host vs. non-host tree species (Campbell and Borden 2005).

In conclusion, we showed that trap color is a key visual cue that can be exploited to develop efficient trapping protocols for longhorn beetles. Different cerambycid subfamilies may require different trap colors; thus, the use of different colored traps can strongly increase the efficiency of the overall surveillance program. Currently, multi-funnel traps or panel traps used for trapping longhorn beetles can be found on the market only in a few colors, i.e., black, green and purple, but our results clearly indicate that more options are needed. In addition, showing that visual stimuli are used by both flower-visiting and non-flower-visiting longhorn beetles, our study opens up a new perspective in this field of research. As recently shown for horseflies (Meglič et al. 2019), a detailed investigation of vision and color perception mechanisms can lead to important improvements in trapping techniques for a given taxon. For longhorn beetles, such an approach could result in benefits for surveillance strategies of exotic and native species moved in trade (Poland and Rassati 2019), management of native and exotic species damaging urban or forest trees (Pawson and Watt 2009; Pawson et al. 2009), and also monitoring strategies for endangered species (Larsson 2016). Future studies should try to investigate to what extent color vision is involved in longhorn beetle mate location, as well as to explore whether males and females show different preferences. In addition, it would be important to test whether trap color can affect other woodboring beetles, such as bark and ambrosia beetles or jewel beetles. This would allow to develop a trapping protocol that can attract several wood-boring beetle species simultaneously, saving resources and improving the overall efficacy of generic surveillance programs.

\section{Author's contribution}

DR, GC, LM and JS conceived the study; DR and GC wrote the manuscript; GC and SM conducted field experiments; DR, GC and LM analyzed the data; MF provided funds; FG identified beetles. All authors contributed to and approved the manuscript.

Acknowledgements We thank Manuel Sancassani, Giacomo Ortis, Giacomo Santoiemma, Greta Quero, Manuel Tolin, Michael Bellardi and Enrico Ruzzier for technical assistance in the field and in the laboratory; "Parco Regionale dei Colli Euganei" for providing permission to carry out the trapping study; Riccardo Poloni for advices on longhorn beetle biological attributes; Robert Haack and two anonymous reviewers for comments and suggestions on an earlier draft of this manuscript.

Funding Open access funding provided by Università degli Studi di Padova within the CRUI-CARE Agreement. "Budget Integrato per la Ricerca dei Dipartimenti (BIRD 2018)" and "Dotazione Ordinaria Ricerca (DOR)," University of Padova.

Availability of data and materials Raw data are available in the Supplementary Materials.

Code availability Not applicable.

\section{Compliance with ethical standards}

Conflicts of interest Authors declare that they have no conflict of interests.

Ethical approval This article does not contain any studies with human participants or vertebrates performed by any of the authors.

Open Access This article is licensed under a Creative Commons Attribution 4.0 International License, which permits use, sharing, adaptation, distribution and reproduction in any medium or format, as long as you give appropriate credit to the original author(s) and the source, provide a link to the Creative Commons licence, and indicate if changes were made. The images or other third party material in this article are included in the article's Creative Commons licence, unless indicated otherwise in a credit line to the material. If material is not included in the article's Creative Commons licence and your intended use is not permitted by statutory regulation or exceeds the permitted use, you will need to obtain permission directly from the copyright holder. To view a copy of this licence, visit http://creativecommons.org/licenses/by/4.0/.

\section{References}

Allen E, Noseworthy M, Ormsby M (2017) Phytosanitary measures to reduce the movement of forest pests with the international trade of wood products. Biol Invasions 19:3365-3376

Allison JD, Redak RA (2017) The impact of trap type and design features on survey and detection of bark and woodboring beetles and their associates: a review and meta-analysis. Annu Rev Entomol 62:127-146

Allison JD, Bhandari BD, McKenney JL, Millar JG (2014) Design factors that influence the performance of flight intercept traps for the capture of longhorned beetles (Coleoptera: Cerambycidae) 
from the subfamilies Lamiinae and Cerambycinae. PLoS ONE 9:e93203

Allison JD, Graham EE, Poland TM, Strom BL (2016) Dilution of fluon before trap surface treatment has no effect on longhorned beetle (Coleoptera: Cerambycidae) captures. J Econ Entomol 109:1215-1219

Allison J, Strom B, Sweeney J, Mayo P (2019) Trap deployment along linear transects perpendicular to forest edges: impact on capture of longhorned beetles (Coleoptera: Cerambycidae). J Pest Sci 92:299-308

Balamurali GS, Edison A, Somanathan H, Kodandaramaiah U (2019) Spontaneous colour preferences and colour learning in the fruitfeeding butterfly, Mycalesis mineus. Behav Ecol Sociobiol 73:39

Bashford R (2008) The development of a port surrounds trapping system for the detection of exotic forest insect pests in Australia. In: Oteng-Amoako AA (ed) New advances and contribution to forestry research. InTech, Rijeka, pp 85-100

Bates D, Maechler M, Bolker B et al. (2017) Linear mixed-effects models using 'Eigen' and S4. R package, version 1.1-15.1-117. https://cran.r-project.org/web/packages/lme4/index.html

Bense U (1995) Longhorn beetles: illustrated key to the Cerambycidae and Vesperidae of Europe. Margraf Verlag, Weikersheim

Bouget C, Brustel H, Brin A, Noblecourt T (2008) Sampling saproxylic beetles with window flight traps: methodological insights. Rev Ecol 63:21-32

Braman SK, Sparks BL, Tedders WL, Mizell RF III, Hudson WG (2003) Effects of trap color and bait type on collection of Coleoptera in pyramid traps in commercial nurseries. J Entomol Sci 38:254-261

Brockerhoff EG, Liebhold AM (2017) Ecology of forest insect invasions. Biol Invasions 19:3141-3159

Brockerhoff EG, Jones DC, Kimberley MO, Suckling DM, Donaldson T (2006) Nationwide survey for invasive wood-boring and bark beetles (Coleoptera) using traps with pheromones and kairomones. For Ecol Manag 228:234-240

Campbell SA, Borden JH (2005) Bark reflectance spectra of conifers and angiosperms: implications for host discrimination by coniferophagous bark and timber beetles. Can Entomol 137:719-722

Campbell SA, Borden JH (2009) Additive and synergistic integration of multimodal cues of both hosts and non-hosts during host selection by woodboring insects. Oikos 118:553-563

Campbell JW, Hanula JL (2007) Efficiency of Malaise traps and colored pan traps for collecting flower visiting insects from three forested ecosystems. J Insect Cons 11:399-408

Canadian Food Inspection Agency (CFIA) (2017) Plant protection survey reports 2017. CFIA, Ottawa. http://publications.gc.ca/site/ eng/9.831610/publication.html. Accessed 26 Feb 2020

Collignon RM, Swift IP, Zou YF, McElfresh JS, Hanks LM, Millar JG (2016) The influence of host plant volatiles on the attraction of longhorn beetles to pheromones. J Chem Ecol 42:215-229

De Groot P, Nott RW (2001) Evaluation of traps of six different designs to capture pine sawyer beetles (Coleoptera: Cerambycidae). Agric For Entomol 3:107-111

Eyre D, Haack RA (2017) Invasive cerambycid pests and biosecurity measures. In: Wang Q (ed) Cerambycidae of the world: biology and pest management. CRC Press, Boca Raton, pp 563-618

Faccoli M, Gatto P (2015) Analysis of costs and benefits of Asian longhorned beetle eradication in Italy. Forestry 89:301-309

Fan JT, Denux O, Courtin C, Bernard A, Javal M, Millar JG, Hanks LM, Roques A (2019) Multi-component blends for trapping native and exotic longhorn beetles at potential points-of-entry and in forests. J Pest Sci 92:281-297

Flaherty L, Gutowski JMG, Hughes C, Mayo P, Mokrzycki T, Pohl G, Silk P, Van Rooyen K, Sweeney J (2019) Pheromone-enhanced lure blends and multiple trap heights improve detection of bark and wood-boring beetles potentially moved in solid wood packaging. J Pest Sci 92:309-325

Fukaya M, Akino T, Yasuda T, Yasui H, Wakamura S (2004) Visual and olfactory cues for mate orientation behaviour in male whitespotted longicorn beetle, Anoplophora malasiaca. Entomol Exp Appl 111:111-115

Fukaya M, Akino T, Yasui H, Yasuda T, Wakamura S, Yamamura K (2005) Effect of size and color of female models for male mate orientation in the white spotted longicorn beetle Anoplophora malasiaca (Coleoptera: Cerambycidae). Appl Entomol Zool 40:513-519

Giurfa M, Nunez J, Chittka L, Menzel R (1995) Colour preferences of flower-naive honeybees. J Comp Physiol A 177:247-259

Graham EE, Poland TM (2012) Efficacy of fluon conditioning for capturing cerambycid beetles in different trap designs and persistence on panel traps over time. J Econ Entomol 105:395-401

Graham EE, Poland TM, McCullough DG, Millar JG (2012) A comparison of trap type and height for capturing cerambycid beetles (Coleoptera). J Econ Entomol 105:837-846

Gumbert A (2000) Color choices by bumble bees (Bombus terrestris): innate preferences and generalization after learning. Behav Ecol Sociobiol 48:36-43

Haack RA (2017) Cerambycid pests in forests and urban trees. In: Wang Q (ed) Cerambycidae of the world: biology and pest management. CRC Press, Boca Raton, pp 351-408

Haack RA, Hérard F, Sun JH, Turgeon JJ (2010) Managing invasive populations of Asian longhorned beetle and citrus longhorned beetle: a worldwide perspective. Annu Rev Entomol 55:521-546

Hanks LM, Millar JG (2016) Sex and aggregation-sex pheromones of cerambycid beetles: basic science and practical applications. J Chem Ecol 42:631-654

Hartig F (2017) Package 'DHARMa' residual diagnostics for hierarchical (multi-level/mixed) regression models. Version 0.1.5.https://cran.r-project.org/web/packages/DHARMa/ DHARMa.pdf.

Humble L (2010) Pest risk analysis and invasion pathways - insects and wood packing revisited: What have we learned? NZ J For Sci 40:S57-S72

Imrei Z, Kovats Z, Toshova TB, Subchev M, Harmincz K, Szarukan I, Domingue MJ, Toth M (2014) Development of a trap combining visual and chemical cues for the alfalfa longhorn beetle, Plagionotus floralis. Bull Insectol 67:161-166

Johnson TD, Hanson E, Yu A (2019) Adults of the cerambycid beetle Megacyllene caryae use both olfactory and visual information to locate mates. Entomol Exp Appl 167:500-506

Kerr JL, Kelly D, Bader MKF, Brockerhoff EG (2017) Olfactory cues, visual cues, and semiochemical diversity interact during host location by invasive forest beetles. J Chem Ecol 43:17-25

Larsson MC (2016) Pheromones and other semiochemicals for monitoring rare and endangered species. J Chem Ecol 42:853-868

Lu W, Wang Q, Tian MY, He XZ, Zeng XL, Zhong YX (2007) Mate location and recognition in Glenea cantor (Fabr.)(Coleoptera: Cerambycidae: Lamiinae): roles of host plant health, female sex pheromone, and vision. Environ Entomol 36:864-870

Lyu F, Hai X, Wang Z, Yan A, Liu B, Bi Y (2015) Integration of visual and olfactory cues in host plant identification by the Asian longhorned beetle, Anoplophora glabripennis (Motschulsky) (Coleoptera: Cerambycidae). PLoS ONE 10:e0142752

Meglič A, Ilić M, Pirih P, Škorjanc A, Wehling MF, Kreft M, Belušič G (2019) Horsefly object-directed polarotaxis is mediated by a stochastically distributed ommatidial subtype in the ventral retina. Proc Natl Acad Sci 116:21843-21853

Meurisse N, Rassati D, Hurley BP, Brockerhoff EG, Haack RA (2019) Common pathways by which non-native forest insects move internationally and domestically. J Pest Sci 92:13-27 
Millar JG, Hanks LM (2017) Chemical ecology of cerambycid beetles. In: Wang Q (ed) Cerambycidae of the world: biology and pest management. CRC Press, Boca Raton, pp 161-208

Miller DR, Crow CM, Mayo PD, Silk PJ, Sweeney JD (2015) Responses of Cerambycidae and other insects to traps baited with ethanol, 2,3-hexanediol, and 3,2-hydroxyketone lures in northcentral Georgia. J Econ Entomol 108:2354-2365

Miller DR, Crowe CM, Sweeney JD (2020) Trap height affects catches of bark and woodboring beetles (Coleoptera: Curculionidae, Cerambycidae) in baited multiple-funnel traps in southeastern United States. J Econ Entomol 113:273-280

Mitchell RF, Curkovic T, Mongold-Diers JA, Neuteboom L, Galbrecht HM, Tröger A, Bergmann J, Francke W, Hanks LM (2017) Evidence that cerambycid beetles mimic vespid wasps in odor as well as appearance. J Chem Ecol 43:75-83

Molander MA, Eriksson B, Winde IB, Zou Y, Millar JG, Larsson MC (2019) The aggregation-sex pheromones of the cerambycid beetles Anaglyptus mysticus and Xylotrechus antilope ssp. antilope: new model species for insect conservation through pheromonebased monitoring. Chemoecology 29:111-124

Monnè ML, Monnè MA, Wang Q (2017) General morphology, classification, and biology of Cerambycidae. In: Wang Q (ed) Cerambycidae of the world: biology and pest management. CRC Press, Boca Raton, pp 1-70

Pawson SM, Watt MS (2009) An experimental test of a visual-based push-pull strategy for control of wood boring phytosanitary pests. Agric For Entomol 11:239-245

Pawson SM, Watt MS, Brockerhoff EG (2009) Using differential responses to light spectra as a monitoring and control tool for Arhopalus ferus (Coleoptera: Cerambycidae) and other exotic wood-boring pests. J Econ Entomol 102:79-85

Poland TM, Rassati D (2019) Improved biosecurity surveillance of non-native forest insects: a review of current methods. J Pest Sci 92:37-49

R Core Team (2019) R: a language and environment for statistical computing. R Foundation for Statistical Computing, Austria

Rassati D, Petrucco Toffolo E, Battisti A, Faccoli M (2012) Monitoring of the pine sawyer beetle Monochamus galloprovincialis by pheromone traps in Italy. Phytoparasitica 40:329-336

Rassati D, Faccoli M, Petrucco Toffolo E, Battisti A, Marini L (2015) Improving the early detection of alien wood-boring beetles in ports and surrounding forests. J Appl Ecol 52:50-58

Rassati D, Marini L, Marchioro M, Rapuzzi P, Magnani G, Poloni R, Di Giovanni F, Mayo P, Sweeney J (2019) Developing trapping protocols for wood-boring beetles associated with broadleaf trees. J Pest Sci 92:267-279

Rassati D, Marchioro M, Flaherty L, Poloni R, Edwards S, Faccoli M, Sweeney J (2020) Response of native and exotic longhorn beetles to common pheromone components provides partial support for the pheromone-free space hypothesis. Insect Sci. https://doi. org/10.1111/1744-7917.12790

Schaefer HM, Schaefer V, Levey DJ (2004) How plant-animal interactions signal new insights in communication. Trends Ecol Evol 19:577-584

Sharkey CR, Fujimoto MS, Lord NP, Shin S, McKenna DD, Suvorov A, Martin JG, Bybee SM (2017) Overcoming the loss of blue sensitivity through opsin duplication in the largest animal group, beetles. Sci Rep 7:1-10

Sheehan TN, Ulyshen MD, Horn S, Hoebeke ER (2019) Vertical and horizontal distribution of bark and woodboring beetles by feeding guild: is there an optimal trap location for detection? J Pest Sci 92:327-341

Shipman NM (2011) Role of color and odor on the attraction of insect visitors to spring blooming Trillium, M.S. thesis, Western Carolina University, Cullowhee, pp. 76

Skvarla MJ, Dowling AP (2017) A comparison of trapping techniques (Coleoptera: Carabidae, Buprestidae, Cerambycidae, and Curculionoidea excluding Scolytinae). J Insect Sci 17:1-28

Skvarla MJ, Holland JD (2011) Nontarget insects caught on emerald ash borer purple monitoring traps in western Pennsylvania. North J Appl For 28:219-221

Streinzer M, Roth N, Paulus HF, Spaethe J (2019) Color preference and spatial distribution of glaphyrid beetles suggest a key role in the maintenance of the color polymorphism in the peacock anemone (Anemone pavonina, Ranunculaceae) in Northern Greece. J Comp Physiol A 205:735-743

Toshova TB, Subchev M, Abaev V, Vuts J, Imrei Z, Koczor S, Galli Z, van de Ven R, Toth M (2016) Responses of Pseudovadonia livida adults to olfactory and visual cues. Bull Insectol 69:161-172

Ulyshen MD, Sheehan TN (2019) Trap height considerations for detecting two economically important forest beetle guilds in southeastern US forests. J Pest Sci 92:253-265

Vuts J, Kaydan MB, Yarimbatman A, Tóth M (2012) Field catches of Oxythyrea cinctella using visual and olfactory cues. Physiol Entomol 37:92-96

Wang Q (2002) Sexual selection of Zorion guttigerum Westwood (Coleoptera: Cerambycidae: Cerambycinae) in relation to body size and color. J Insect Behav 15:675-687

Wu Y, Trepanowski NF, Molongoski JJ, Reagel PF, Lingafelter SW, Nadel H, Myers SW, Ray AM (2017) Identification of wood-boring beetles (Cerambycidae and Buprestidae) intercepted in trade associated solid wood packaging material using DNA barcoding and morphology. Sci Rep 7:40316

Publisher's Note Springer Nature remains neutral with regard to jurisdictional claims in published maps and institutional affiliations. 